\title{
EFFECTS OF OVERALL LOW-BEAM INTENSITY ON SEEING DISTANCE IN THE PRESENCE OF GLARE
}

\author{
Michael J. Flannagan \\ Michael Sivak \\ Eric C. Traube \\ Shinichi Kojima
}

July 1996 


\title{
EFFECTS OF OVERALL LOW-BEAM INTENSITY ON SEEING DISTANCE IN THE PRESENCE OF GLARE
}

\author{
Michael J. Flannagan \\ Michael Sivak \\ Eric C. Traube \\ Shinichi Kojima
}

The University of Michigan

Transportation Research Institute

Ann Arbor, Michigan 48109-2150

U.S.A.

Report No. UMTRI-96-26

July 1996 
Technical Report Documentation Page

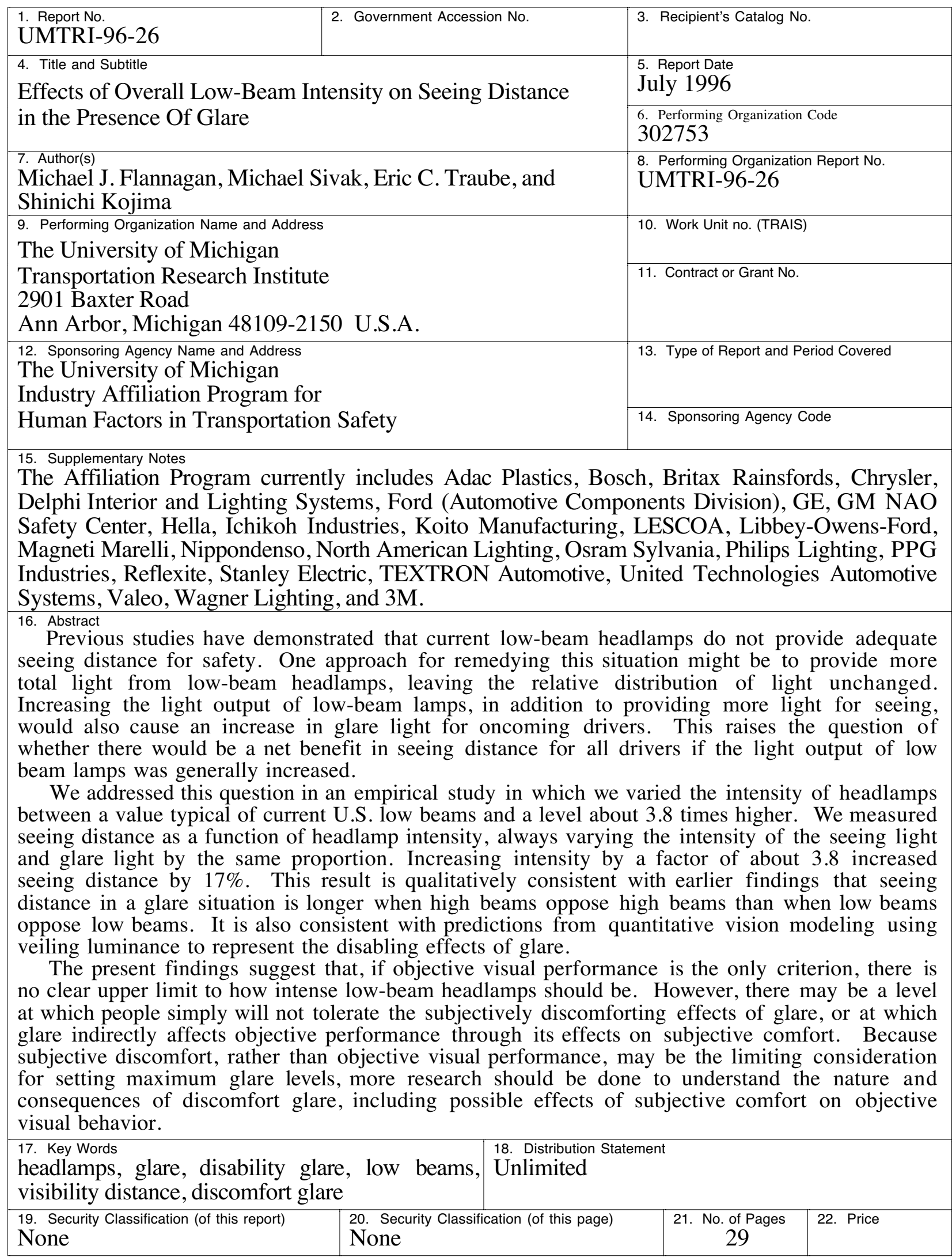




\section{Acknowledgments}

Appreciation is extended to the members of the University of Michigan Industry Affiliation Program for Human Factors in Transportation Safety for support of this research. The current members of the Program are:

Adac Plastics

Bosch

Britax Rainsfords

Chrysler

Delphi Interior and Lighting Systems

Ford (Automotive Components Division)

GE

GM NAO Safety Center

Hella

Ichikoh Industries

Koito Manufacturing

LESCOA

Libbey-Owens-Ford

Magneti Marelli

Nippondenso

North American Lighting

Osram Sylvania

Philips Lighting

PPG Industries

Reflexite

Stanley Electric

TEXTRON Automotive

United Technologies Automotive Systems

Valeo

Wagner Lighting

$3 \mathrm{M}$

We thank Walter Kosmatka of GE Lighting for photometering the lamps used in this study. 


\section{Contents}

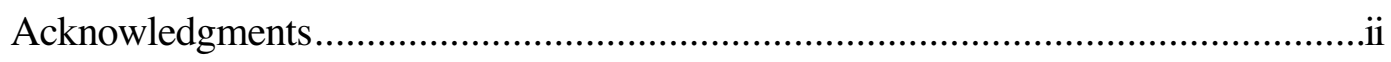

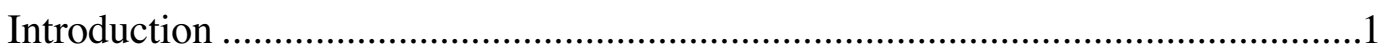

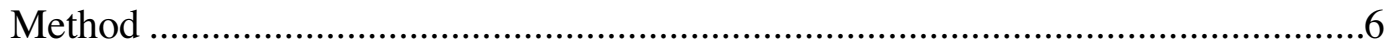

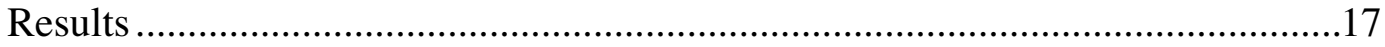

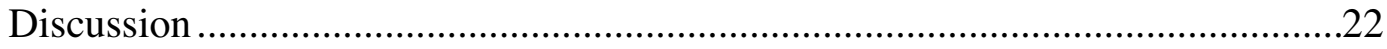

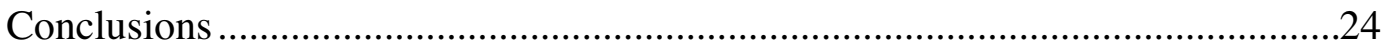

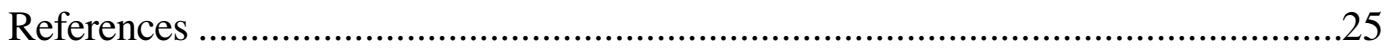




\section{Introduction}

Previous studies have demonstrated that current low-beam headlamps do not provide adequate seeing distance for safety (e.g., Sivak, Helmers, Owens, \& Flannagan, 1992). This problem is partly due to the fact that, by definition, low-beam patterns are designed by biasing the distribution of light away from the straight ahead in order to achieve a desirable compromise between providing seeing light for the driver using the lamps, and reducing glare light to oncoming drivers. There has been much discussion of the relative merits of the various strategies for distributing low-beam light, including attempts to harmonize the currently differing approaches in Europe and the U.S. (Sivak \& Flannagan, 1994).

In the present research we evaluated an approach to improving low-beam performance that is partly independent of the issue of how light should be distributed: increasing the overall light output of low-beam lamps. Although, as we will discuss later, certain considerations limit the practical value of this approach as a solution to the problem of inadequate low-beam seeing distance, the results reported here illustrate an important difference between how glare affects objective seeing ability and how it affects subjective comfort. We suggest that there should be further research and discussion about the implications of this difference for low-beam design.

Could current low-beam performance be improved by providing more total light without changing the relative distribution of the light? Increasing the light output of lowbeam lamps, in addition to providing more light for seeing, would also cause an increase in glare light for oncoming drivers. This raises the question of whether there would be a net benefit in seeing distance for all drivers if the light output of low-beam lamps was generally increased. Would the benefit of more seeing light outweigh the negative effects of glare?

This question was directly addressed by an empirical study in which we varied the intensity of headlamps between a value typical of current U.S. low beams and a level about 3.8 times higher. Our experimental situation involved two identical sets of headlamps, one to provide light for seeing, and an opposing set that provided glare light. We measured seeing distance as a function of headlamp intensity, always varying the intensity of the seeing light and glare light by the same proportion. Neutral density filters were used to vary the output of the lamps so intensity could be manipulated while keeping the beam patterns of both the seeing and glare lamps unchanged.

Before reporting the new experimental results, we review two other sources of information that bear on the question of how overall intensity affects seeing in the presence of glare. First, we summarize the findings of several previous studies that measured seeing distance for low beams opposing low beams, and high beams opposing high beams. 
Second, we review the predictions of quantitative modeling of stimulus visibility for a situation in which seeing light and glare light are increased proportionately.

\section{Comparisons of low and high beams}

A series of studies in Sweden (Johansson, Bergstörm, et al., 1963; Johansson \& Rumar, 1963) measured seeing distances in meeting situations in which the lamps of the meeting vehicles were either both low beams or both high beams. The results indicated that, over a wide range of conditions, seeing distances were longer when both vehicles were using high beams. Johansson, Bergstörm, et al. summarized the results of one experiment as follows:

It was assumed, as a starting point for this experiment, that...full headlights allow longer visible distance than dipped lights when the distance between cars is large, e.g. more than 1000 metres, and that dipped lights allow longer visible distance when the distance between the cars is small....

The results of this preliminary experiment showed, contrary to expectation, that full headlights gave visible distances at least as great if not greater than those given by dipped lights for the whole course of the meeting. [emphasis in original] (p. 173)

These results bear on the topic of the present study (effects of increased total light with a fixed, low-beam distribution) because the shift from low to high beams increases both the light on stimuli down the road and the glare to opposing drivers. Although these results suggest that proportional increases in glare and seeing light may lead to better seeing distances, they cannot be considered conclusive because comparisons of high and low beams also involve large changes in beam patterns.

\section{Predictions based on vision modeling}

Visual performance is a complex process that is affected by many factors - the age and visual acuity of the observer, the size and duration of the stimulus, to mention just a few. The difficulty of making predictions about visual performance is indicated in part by the size and complexity of the two volumes of Publication 19/2 of the Commission Internationale de l'Eclairage (CIE), which describe a quantitative model for the visibility of stimuli (CIE, 1981a, 1981b). However, in spite of the complexity of that model, its general approach can be used rather simply to generate a prediction about the effects of low-beam intensity with reasonable confidence. The key parameters of the model are the luminance of 
the background against which a stimulus is presented, $L_{b}$, and the luminance contrast between the stimulus and that background:

$$
C=\frac{L_{s}-L_{b}}{L_{b}}
$$

where $L_{s}$ is the luminance of the stimulus. A set of empirical functions such as the one shown in Figure 1, representing the contrast required at a given background luminance for a stimulus to reach a certain criterion of visibility, is used in the model to generate predictions about the visibility of stimuli under a wide range of conditions. The function in Figure 1, for example, is for a round stimulus with a diameter of 4 minutes of arc, exposed for 0.2 seconds, and viewed foveally by people between 20 and 30 years of age.

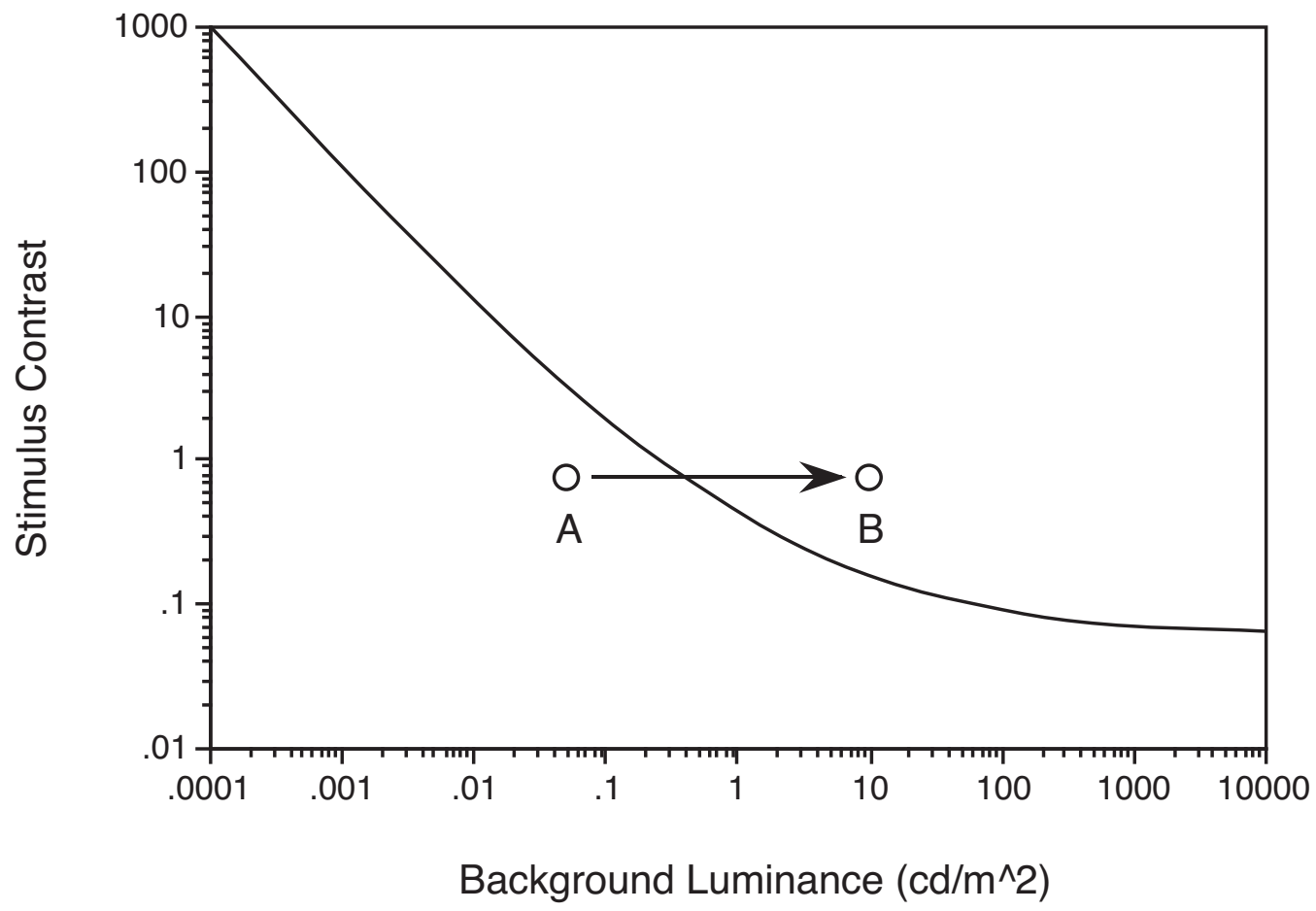

Figure 1. The curved line shows threshold contrast for visual detection as a function of background luminance. Adapted from Figure 3 in Volume 1 of CIE Publication 19/2 (CIE, 1981a, page 9). Combinations of contrast and background that correspond to points below the function are below visual threshold, and combinations that fall above the function are above threshold. See text for explanation of the points marked A and B.

An important characteristic of the function in Figure 1 turns out to be true for all such functions: they are monotonically decreasing (at least up to a reasonably high background luminance, well above the levels of concern in night driving). It is this 
characteristic that leads to a simple and general prediction about the effects of increased low-beam intensity.

The disabling effects of glare can be represented in the model in terms of a veiling luminance, $L_{v}$. This veiling luminance is caused by scattering of light from the glare source. The scattering may occur in the eye itself, or as a result of something between the observer and the stimulus, such as dirt on a windshield or raindrops in the air. The veiling luminance will appear to the observer to be added to both the luminance of the stimulus and the luminance of the background. The contrast value incorporating the effects of glare, $C_{g}$, will therefore be:

$$
C_{g}=\frac{\left(L_{s}+L_{v}\right)-\left(L_{b}+L_{v}\right)}{L_{b}+L_{v}}=\frac{L_{s}-L_{b}}{L_{b}+L_{v}}
$$

Because the veiling luminance is effectively added only to the denominator of this expression, the effects of glare will always be to reduce contrast.

In driving situations in which ambient illumination is small relative to headlamp illumination, such as at night on roads without fixed lighting, the luminances of the stimulus and of the background will be directly proportional to the intensity of the lamps on the observer's vehicle, and the veiling luminance will be directly proportional to the intensity of the lamps on the opposing (glare) vehicle. Therefore, if both of those sets of lamps are changed by a common factor, $\alpha$, the new value of contrast, $C_{g}$, will be:

$$
C_{g}^{\prime}=\frac{\alpha L_{s}-\alpha L_{b}}{\alpha L_{b}+\alpha L_{v}}=\frac{L_{s}-L_{b}}{L_{b}+L_{v}}=C_{g}
$$

Thus, contrast will be unaffected when the seeing lamps and glare lamps are changed by the same factor. However, the new value of the other key parameter, background luminance, will now be:

$$
L_{b}^{\prime}=\alpha L_{b}
$$

For example, if $\alpha$ is greater than 1.0 the new background luminance will be higher. Such an increase in intensity might be represented by the shift from point A to point B in Figure 1. The shift is horizontal because the value on the vertical axis, representing contrast, is unchanged. In this example, the shift moves the stimulus from the region below visual 
threshold into the region above visual threshold. Thus, a stimulus that was not visible can become visible with a general increase in lamp intensity.

Not all general increases in illumination will cause a stimulus to cross a visibility threshold. In some cases, increases in illumination will cause shifts within the visible region or within the nonvisible region of a graph such as the one in Figure 1. However, because general increases in intensity can only shift stimulus points to the right along a horizontal line, and because the functions representing visual thresholds are monotonically decreasing, increases in illumination can never shift stimuli from a visible region into a nonvisible region. This is true even though this analysis accounts for the disabling effects of increased glare light. According to this model of vision, general increases in light intensity will sometimes improve visual performance, and will never diminish it. 


\section{Method}

\section{Subjects}

Fifteen paid subjects participated in this study. There were eight younger subjects (ranging from 20 to 27 years old, with a mean of 22.1), and seven older subjects (ranging from 65 to 78, with a mean of 72.7). In the younger group there were five males and three females; in the older group there were three males and four females. All subjects were licensed drivers.

\section{Experimental Lighting Equipment}

Two identical lamp racks were constructed, one to provide seeing light and one to provide glare light. Each rack held eight low-beam headlamps, in two clusters of four. The centers of the clusters were mounted $0.6 \mathrm{~m}$ above the ground and the centers of the clusters were $1.2 \mathrm{~m}$ apart horizontally. These distances are typical of standard low-beam headlamp systems. Figure 2 shows the configuration of one of the experimental headlamp racks, and the geometry of a typical standard headlamp system on which it was based. All eight lamps were rectangular, low-beam, tungsten-halogen lamps (2A1, 100 by $165 \mathrm{~mm})$. The beam patterns of all lamps were similar; an isocandela diagram for one of the lamps is shown in Figure 3.

The clustering of the headlamps reduced parallax effects so that light output of each cluster would closely approximate the light distribution that would have resulted from a single headlamp of 3.8 times normal intensity. The parallax between the center of any of the four headlamps in a cluster and the center of the cluster itself (corresponding to the center of a standard single headlamp that the cluster was designed to mimic) was only 0.1 degree for a point $50 \mathrm{~m}$ from the lamps (a typical seeing distance in the study).

All headlamps were visually aimed so that their high intensity zones were just below and to the right of the straight ahead (normal aiming for U.S. low beams). The aiming screen was adjusted for the height and lateral position of each member of the clusters of four lamps, so that the optical axes of the four lamps would be parallel (rather than converging at some distance). 

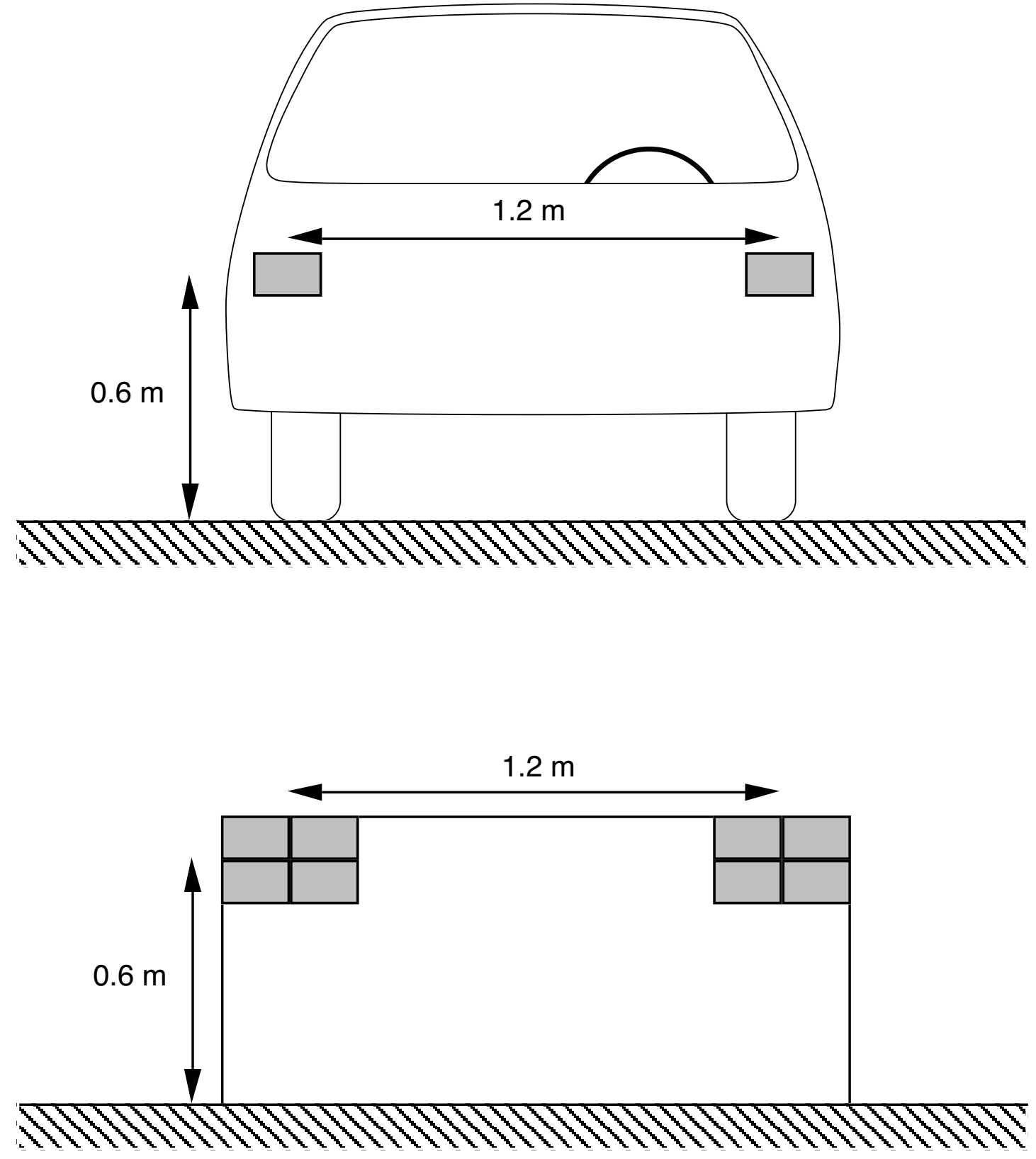

Figure 2. The geometry of the eight-headlamp system (lower panel) and the geometry of a typical standard headlamp system (with 0.6-meter mounting height and 1.2-meter spacing) on which it was based (upper panel). Each gray rectangle represents one headlamp. Each four-headlamp cluster was centered on the position of one lamp in the normal headlamp system. 


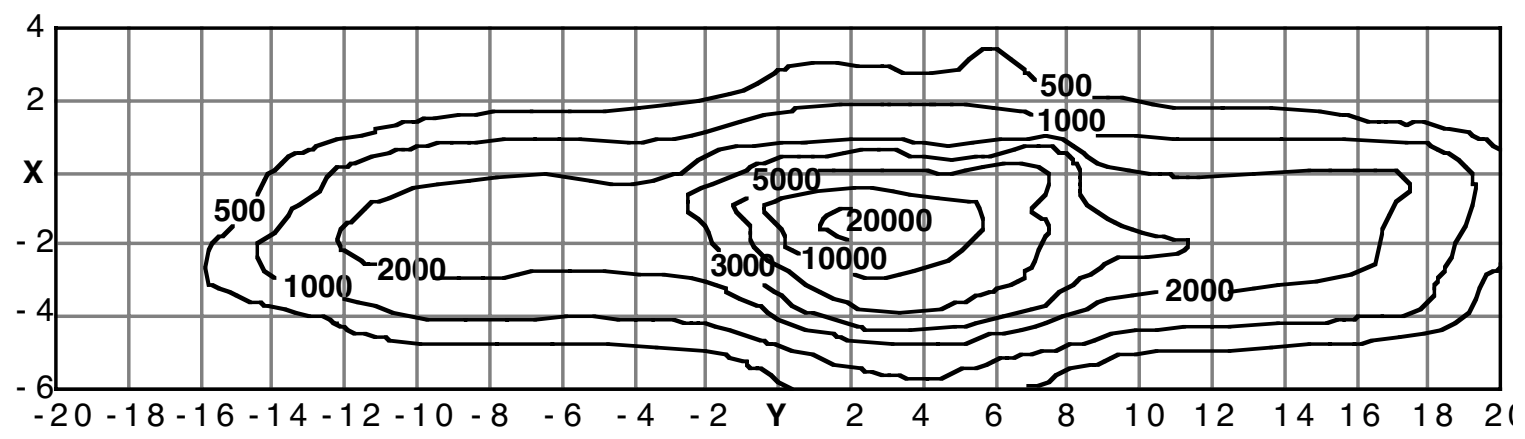

Figure 3. The isocandela diagram for one of the headlamps used in the eight-headlamp racks. (All lamps were similar.) The numbers labeling the contours are in candelas, and the numbers on the axes are in degrees.

Each of the two lamp racks was equipped with three pairs of neutral density filters that could be individually mounted in front of headlamp clusters, completely covering them. The nominal densities and transmittances of the filters are given in Table 1. The lightest filters had little effect on light output, leaving the output of the experimental lamp systems at almost four times the output of a single standard low-beam lamp system. These filters were used, rather than simply leaving the lamps unfiltered, to insure that any scattering effects of dirt on the filters, or of the filter material itself, were approximately equal across lighting conditions. The two denser filters were selected to reduce the output of the experimental lamp systems by factors of approximately two and four. Thus, with the densest filter the light output was about the same as that of a standard low-beam headlamp system. The headlamp racks were powered by precision power supplies set at 12.8 volts.

Table 1

Nominal densities and transmittances of the three pairs of filters used with the experimental lamp racks. Actual photometric values measured in the field are given in Tables 5, 6, and 7 .

\begin{tabular}{|c|c|}
\hline Density & Transmittance \\
\hline 0.022 & 0.95 \\
0.3 & 0.50 \\
0.6 & 0.25 \\
\hline
\end{tabular}




\section{Test site}

The experiment was conducted on a straight, level section of a two-lane access road near the University of Michigan Transportation Research Institute that was closed to traffic during testing (see Figure 4). The pavement surface was dry asphalt. Each lane was 12 feet $(3.7 \mathrm{~m})$ wide, and the two opposing lamp racks were offset laterally by that distance, as shown in Figure 4.

A car for the subjects to sit in during the experiment was placed on the road as shown in Figure 4. One of the lamp racks was placed in front of it, so that the headlamps on the rack were just in front of the headlamps of the car itself. (The standard headlamps of the car were not used; they remained off throughout the study.) The other lamp rack was placed $30 \mathrm{~m}$ from the subject's eyepoint, facing toward the subject to provide glare similar to that from an opposing vehicle.

Along the right shoulder of the road (from the subject's point of view), distances in front of the car were marked every 2 meters from 30 to 106 meters. (Preliminary tests had indicated that under these specific conditions a pedestrian would always be visible at $30 \mathrm{~m}$ and would never be visible at $106 \mathrm{~m}$.) The marks were on small pieces of tape flush with the surface of the road. They could be read easily by a pedestrian standing on the road near them, but they were not visible at night from the eye position of a driver of the car.

Marks on the pavement defined two paths along which a pedestrian experimenter could walk in approaching and receding from the subject's car. One path ("center") was directly in front of the subject, $0.4 \mathrm{~m}$ to the left (from the subject's point of view) of the middle of the lane that the subject's car was in. The other path ("right") was along the right shoulder, $1.85 \mathrm{~m}$ to the right of the middle of the lane. The visual angle between the glare source and the pedestrian was therefore smaller when the pedestrian approached and receded along the center path. The visual angles (from the subject's point of view) between the pedestrian and the middle of the glare-lamp rack are given in Table 2 for the nearest point $(30 \mathrm{~m})$, a typical seeing distance $(50 \mathrm{~m})$, and the farthest point $(106 \mathrm{~m})$ along the pedestrian paths. Note that the visual angle does not change as the pedestrian walks along the center path, directly toward or away from the subject. 


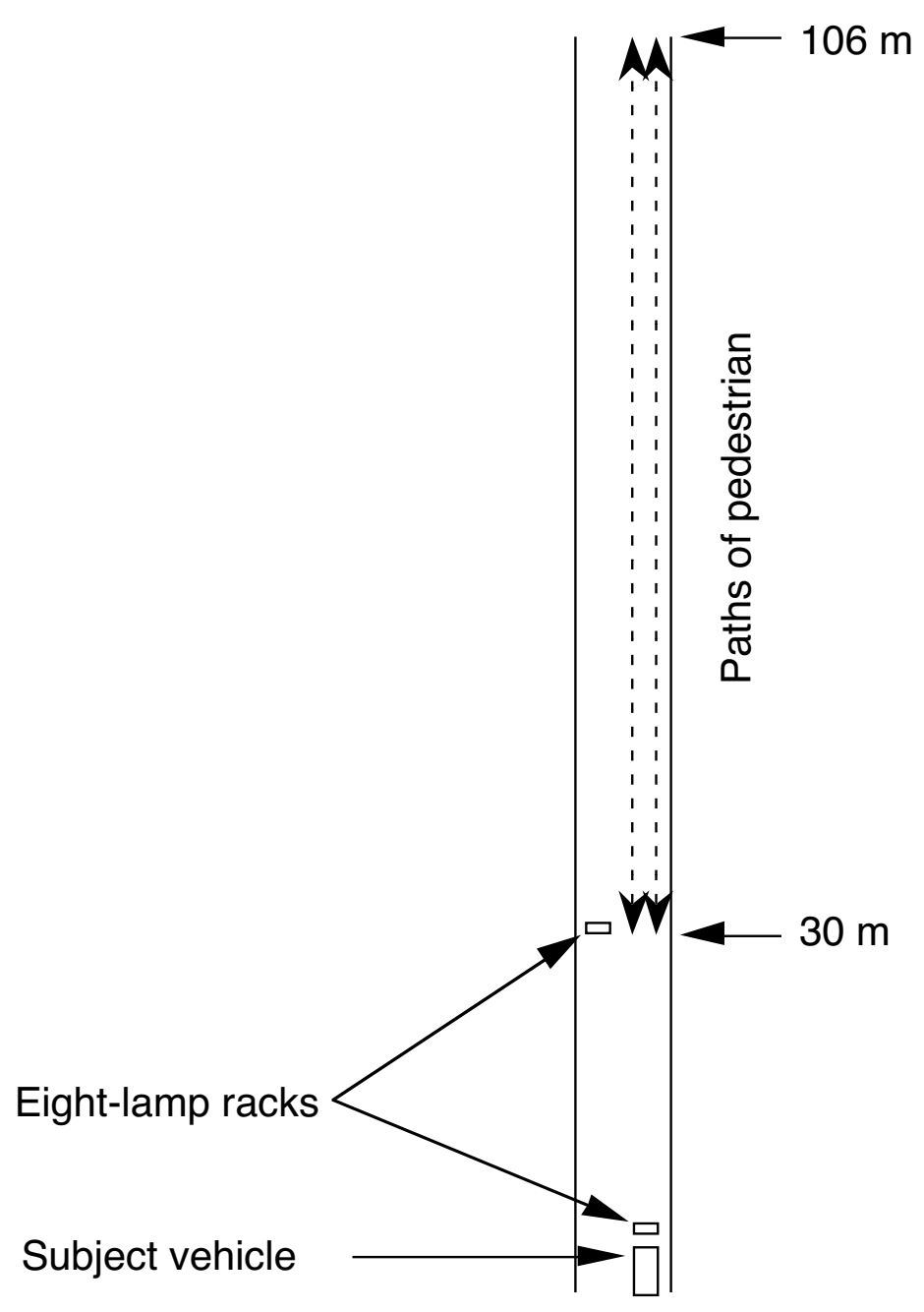

Figure 4. A diagram of the test site showing the positions of the two lamp racks, the car that the subject sat in, and the paths that the pedestrian walked along. One path was directly in front of the subject and the other was along the right shoulder. Distances and sizes are approximately to scale.

Table 2

Visual angle (degrees) between the center of the glare source and the pedestrian for several distances along the two pedestrian paths.

\begin{tabular}{|c|cc|}
\hline \multirow{2}{*}{ Distance to pedestrian (m) } & \multicolumn{2}{|c|}{ Pedestrian path } \\
\cline { 2 - 3 } & Center (in front of subject) & Right (along shoulder) \\
\hline 30 & 6.3 & 10.6 \\
50 & 6.3 & 8.9 \\
106 & 6.3 & 7.5 \\
\hline
\end{tabular}




\section{Lighting conditions}

Three lighting conditions, shown in Table 3, were used during the portion of the experiment in which seeing distance was measured. The neutral density filters were used to produce three levels of illumination from the experimental lamp racks. As indicated in Table 3, the glare and seeing lamps were always varied proportionately.

The same three lighting conditions were used in the portion of the experiment that measured discomfort glare, but they were supplemented with two more conditions in which the seeing light was at the lowest level (which corresponded approximately to standard low beams). This allowed us to measure whether the subjective effects of glare were affected by the seeing lamps. The five lighting conditions used in the discomfort glare portion of the study are shown in Table 4. Discomfort glare could be measured as a function of glarelamp intensity over a set of three conditions in which seeing-lamp intensity was varied proportionately, and over a partially overlapping set of three conditions in which seeinglamp intensity was constant. 
Table 3

The combinations of seeing-lamp and glare-lamp intensities used in the seeing-distance portion of the study. Differences in the intensities of the seeing and glare lamps across conditions were always proportional.

\begin{tabular}{|c|c|c|c|c|}
\cline { 3 - 5 } \multicolumn{2}{c|}{} & \multicolumn{3}{c|}{ Glare lamps } \\
\cline { 2 - 5 } \multicolumn{1}{c|}{} & Low & Medium & High \\
\hline \multirow{3}{*}{ Seeing lamps } & Low & $\mathrm{x}$ & & \\
\cline { 2 - 5 } & Medium & & $\mathrm{x}$ & \\
\cline { 2 - 5 } & High & & & $\mathrm{x}$ \\
\hline
\end{tabular}

Table 4

The combinations of seeing-lamp and glare-lamp intensities used in the discomfort glare portion of the study. The combinations used included both a set in which the intensities of the seeing and glare lamps were varied proportionately, and also a partially overlapping set in which all the glare lamp intensities were paired with the lowest level of the seeing lamps.

\begin{tabular}{|c|c|c|c|c|}
\cline { 3 - 5 } \multicolumn{2}{c|}{} & \multicolumn{3}{c|}{ Glare lamps } \\
\cline { 2 - 5 } \multicolumn{2}{c|}{} & Low & Medium & High \\
\hline \multirow{3}{*}{ Seeing lamps } & Low & $\mathrm{x}$ & $\mathrm{x}$ & $\mathrm{x}$ \\
\cline { 2 - 5 } & Medium & & $\mathrm{x}$ & \\
\cline { 2 - 5 } & High & & & $\mathrm{x}$ \\
\hline
\end{tabular}




\section{Photometry}

Illuminance values for a vertical surface, $0.75 \mathrm{~m}$ above the ground, at distances of 30 , 50 , and $70 \mathrm{~m}$ from the subject along the two pedestrian paths, are given in Table 5 for each of the three lighting conditions used in the seeing-distance task. These readings were taken with both seeing and glare lamps on.

Illuminance values were low (approximately 0.14 lux for all positions, mostly from distant street lamps) when all of the headlamps were off.

Illuminance of the subject's eyepoint (including the influence of the windshield) under the three lighting conditions used in the seeing-distance task, and the additional two lighting conditions used in the discomfort glare rating task, is shown in Table 6.

Luminance of the pavement directly in front of the subject, at distances of 10 and $20 \mathrm{~m}$, measured from the subject's eyepoint (including the influence of the windshield), is shown in Table 7 . These readings were taken using a spot photometer with a 1-degree field of view.

Table 5

Illuminance values (lux) measured on a vertical surface ( $0.75 \mathrm{~m}$ above the ground) at various distances from the subject along the two paths of the pedestrian.

\begin{tabular}{|c|ccc|cccc|}
\hline & \multicolumn{3}{|c|}{ Center path } & \multicolumn{3}{c|}{ Right path } \\
\cline { 2 - 7 } Distance (m) & Low & Medium & High & Low & Medium & High \\
\hline 30 & 4.51 & 8.79 & 15.5 & 5.07 & 9.9 & 17.4 \\
50 & 1.78 & 3.56 & 6.18 & 1.78 & 4.01 & 6.88 \\
70 & 1.26 & 2.43 & 4.03 & 1.26 & 2.46 & 4.31 \\
\hline
\end{tabular}


Table 6

Illuminance values at the subject's eyepoint under the various lighting conditions used in the seeing-distance and discomfort glare tasks.

\begin{tabular}{|c|c|c|}
\hline \multicolumn{2}{|c|}{ Lighting condition } & \multirow[b]{2}{*}{ Illuminance (lux) } \\
\hline Seeing lamps & Glare lamps & \\
\hline Low & Low & 1.23 \\
\hline Low & Medium & 2.35 \\
\hline Low & High & 3.89 \\
\hline Medium & Medium & 2.42 \\
\hline High & High & 4.05 \\
\hline
\end{tabular}

Table 7

Pavement luminances, measured from the subject's eyepoint, for 1-degree fields, at two distances from the subject along a line directly in front of the subject, under the various lighting conditions used in the seeing-distance and discomfort glare tasks.

\begin{tabular}{|cc|cc|}
\hline \multicolumn{2}{|c|}{ Lighting condition } & \multicolumn{2}{c|}{ Luminance $\left(\mathrm{cd} / \mathrm{m}^{2}\right)$} \\
\hline Seeing lamps & Glare lamps & At $10 \mathrm{~m}$ & At $20 \mathrm{~m}$ \\
\hline Low & Low & 0.827 & 0.929 \\
Low & Medium & 0.826 & 1.529 \\
Low & High & 1.009 & 2.497 \\
Medium & Medium & 1.694 & 2.021 \\
High & High & 2.846 & 3.567 \\
\hline
\end{tabular}




\section{Procedure}

General. Subjects were run individually. Each subject sat in the driver's seat of a stationary car (located as shown in Figure 4) throughout his or her participation. One experimenter sat in the subject's car to give the subject instructions, and to signal the other experimenters by radio when each trial was about to begin. Data collection for each subject took about 25 minutes. Subjects sat in the car and adapted to the night lighting conditions for about 10 minutes before data collection began.

All testing was done at night, beginning about an hour after sunset. The moon was below the horizon during testing. There was no fixed lighting near the test site.

The experiment consisted of two phases. The first measured the distance at which the subject could see a pedestrian in front of the car. The second phase measured the discomfort that the subject experienced from the glare of the opposing headlamps.

Seeing distance. In the seeing-distance part of the experiment the subject's task was to look down the road and indicate when a pedestrian walking along the road first became visible (while approaching out of the darkness) or invisible (while receding into the darkness). Subjects were instructed to look straight down the road (i.e., slightly to the right of the glare source). They continued to look down the road in between trials.

On each trial an experimenter serving as the pedestrian started walking toward the subject's position from a point $106 \mathrm{~m}$ away. (The starting point was beyond the seeing distance of any subject in any lighting condition.) The subject indicated when he or she could first see the pedestrian by tapping the car's horn. The pedestrian experimenter noted his position to the nearest meter by looking at the distance marks on the side of the road when he heard the horn, but continued walking until he reached a point $30 \mathrm{~m}$ from car. He then turned around and began walking away along the same path. The subject tapped the horn again when the pedestrian just disappeared. The pedestrian again noted his position and continued walking until he reached the distant starting point.

The pedestrian walked along the same path (center or right) for both the approaching and receding passes of a trial. Because the path was varied randomly from trial to trial, the subject did not know exactly where the pedestrian would appear on approaching passes. The pedestrian wore dark clothing and shoes, all with reflectance of about $5 \%$.

Lighting conditions remained the same during each trial (i.e., an approaching pass combined with a receding pass). Two experimenters, one stationed at the glare-lamp rack and one stationed at the seeing-lamp rack, changed filters between trials according to a sequence that was randomized for each subject. As soon as the subject had tapped the horn on the receding pass, those experimenters changed the filters for the next trial. 
Each subject had 12 seeing-distance trials (each involving two judgments, one as the pedestrian approached and one as the pedestrian receded). There were six different conditions, consisting of the combinations of three lighting conditions (low, medium, and high intensities of the lamps) and two pedestrian paths (center or right). Each condition occurred once in the first six trials, and once in the second six trials. Within each set of six, the order of trials was randomized. On every trial the intensity levels of the seeing and glare lamps were matched, as indicated in Table 3.

Discomfort glare. The discomfort glare part of the experiment immediately followed the seeing-distance part. During the discomfort glare part, the subject's task was to estimate the discomfort he or she experienced from the opposing lamps using the DeBoer scale. The DeBoer scale has been used extensively to evaluate glare in night driving situations (e.g., Bhise, Swigart, \& Farber, 1975; DeBoer, 1967). It is a 9-point scale with qualifiers only for the odd points as follows:

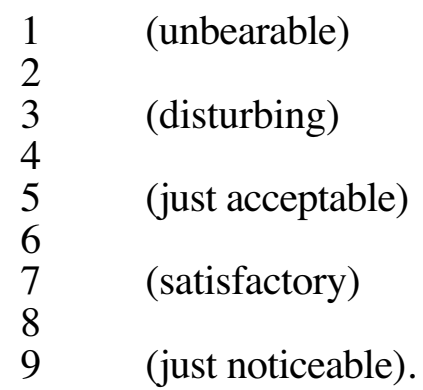

Each discomfort glare trial began with the subject looking down at the instrument panel inside the car and shielding his or her eyes from the scene outside. The glare lamps and the seeing lamps were covered with filters appropriate for the conditions of that trial. When the experimenter inside the car signaled the beginning of the trial the subject raised his or her head and looked down the road directly in front of the car (slightly to the right of the glare source). After a few seconds, the subject made a numerical rating of discomfort glare and then again lowered his or her head. The experimenters then changed the filters for the next trial. The pedestrian was not present during discomfort glare measurements.

Each subject had 12 discomfort glare trials. The first six trials and the second six trials each consisted of the five lighting conditions indicated in Table 4, with the combination of low seeing lamps and low glare lamps occurring twice (because it was considered to be a member of both the seeing-lamps-same and the seeing-lamps-always-low series - see Table 4). Within each set of six, the order of trials was randomized. 


\section{Results}

\section{Seeing distance}

The result of most interest is the main effect of lamp intensity, shown in Figure 5. It was highly significant, $F(2,26)=147.6, p<.0001$. When glare and seeing light are increased proportionately, seeing distance increases by about $17 \%$ over the range of intensities used here (from $47.9 \mathrm{~m}$ in the low condition to $56.0 \mathrm{~m}$ in the high condition).

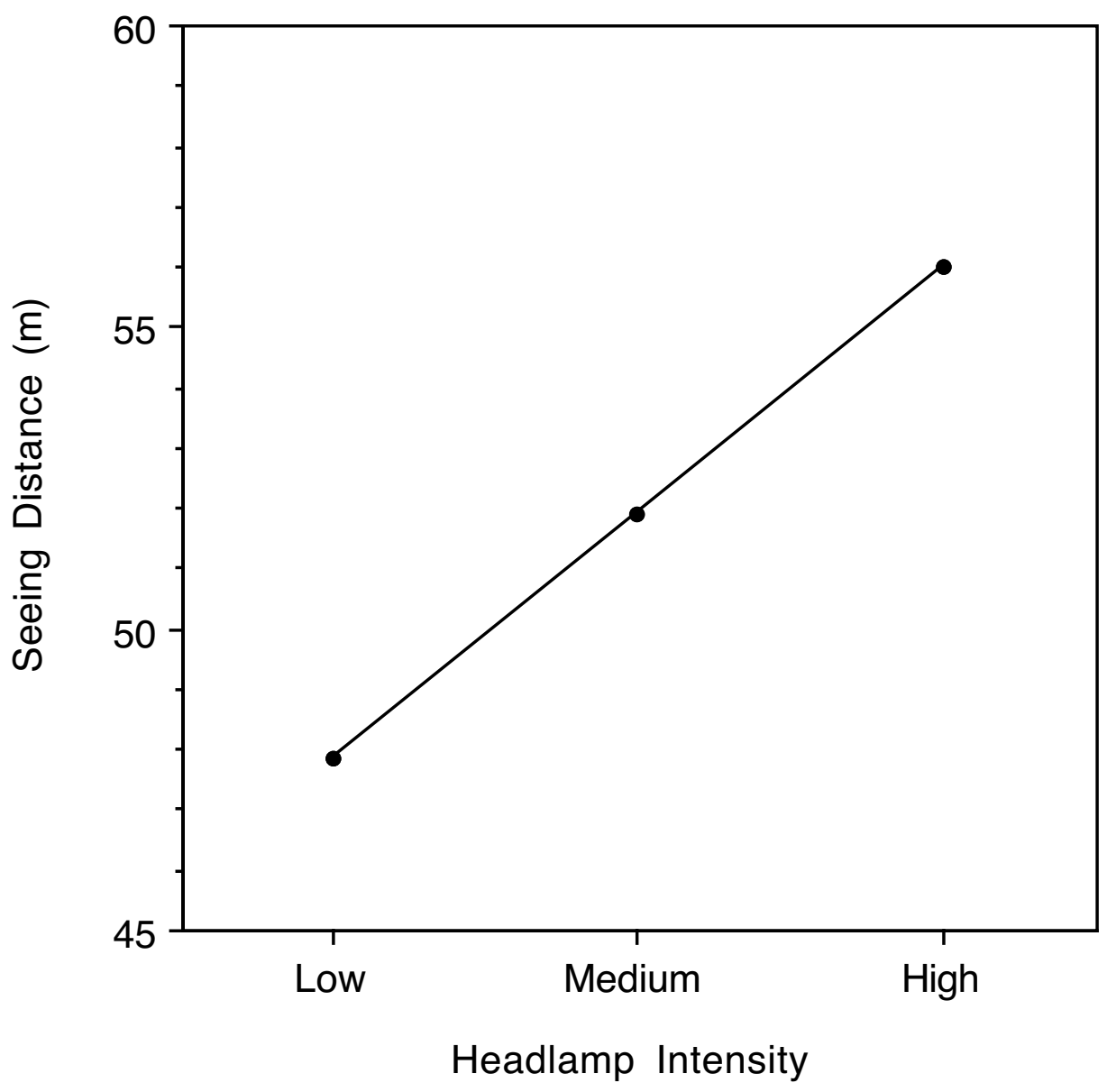

Figure 5. The main effect of headlamp intensity on seeing distance. Seeing lamps and glare lamps were always changed proportionately. 
The path that the pedestrian walked on had a significant effect on seeing distance $F(1,13)=82.7, p<.0001$, with seeing distances being $13 \%$ longer when the pedestrian was on the right path $(55.0 \mathrm{~m})$ than when he was on the center path $(48.8 \mathrm{~m})$. The combined effects of headlamp intensity and the path that the pedestrian was walking on are shown in Figure 6. The effect of intensity on seeing distance was about the same for both paths and the interaction of these variables was not significant, $F(2,26)=0.19, p=.83$. When the pedestrian was on the right path he was always further from the glare source in terms of visual angle seen from the subject's position than when he was on the center path (see Table 2 ). Thus, the greater seeing distances on the right path are consistent with the wellestablished finding that disability glare effects fall off sharply as the distance between the glare source and the stimulus increases (Holladay, 1926; Holladay, 1927). The fact that greater lamp intensity results in longer seeing distances for both paths, in spite of the overall difference in disability glare effects between the paths, indicates that the beneficial effect of greater intensity is reasonably general.

Seeing distance was significantly affected by the direction the pedestrian was walking, $F(1,13)=26.3, p=.0002$, with average seeing distance being shorter when the pedestrian was approaching $(49.1 \mathrm{~m})$ than when he was receding $(54.8 \mathrm{~m})$. This difference may be due to the fact that on approaching trials the subject was not sure which path the pedestrian was on, or exactly when he would appear. In contrast, on receding trials the subject could see the pedestrian clearly when he began walking away.

Subject age group had a significant effect on seeing distance $F(1,13)=5.73$, $p=.032$, with seeing distances being $23 \%$ longer for the younger group $(57.0 \mathrm{~m})$ than for the older group (46.2 m). The combined effects of headlamp intensity and subject age are shown in Figure 7. The interaction of these variables was significant, $F(2,26)=9.45, p=$ .0008. As can be seen in Figure 7, the nature of the interaction appears to be that the effect of intensity for each age group is proportional to that group's average seeing distance. 


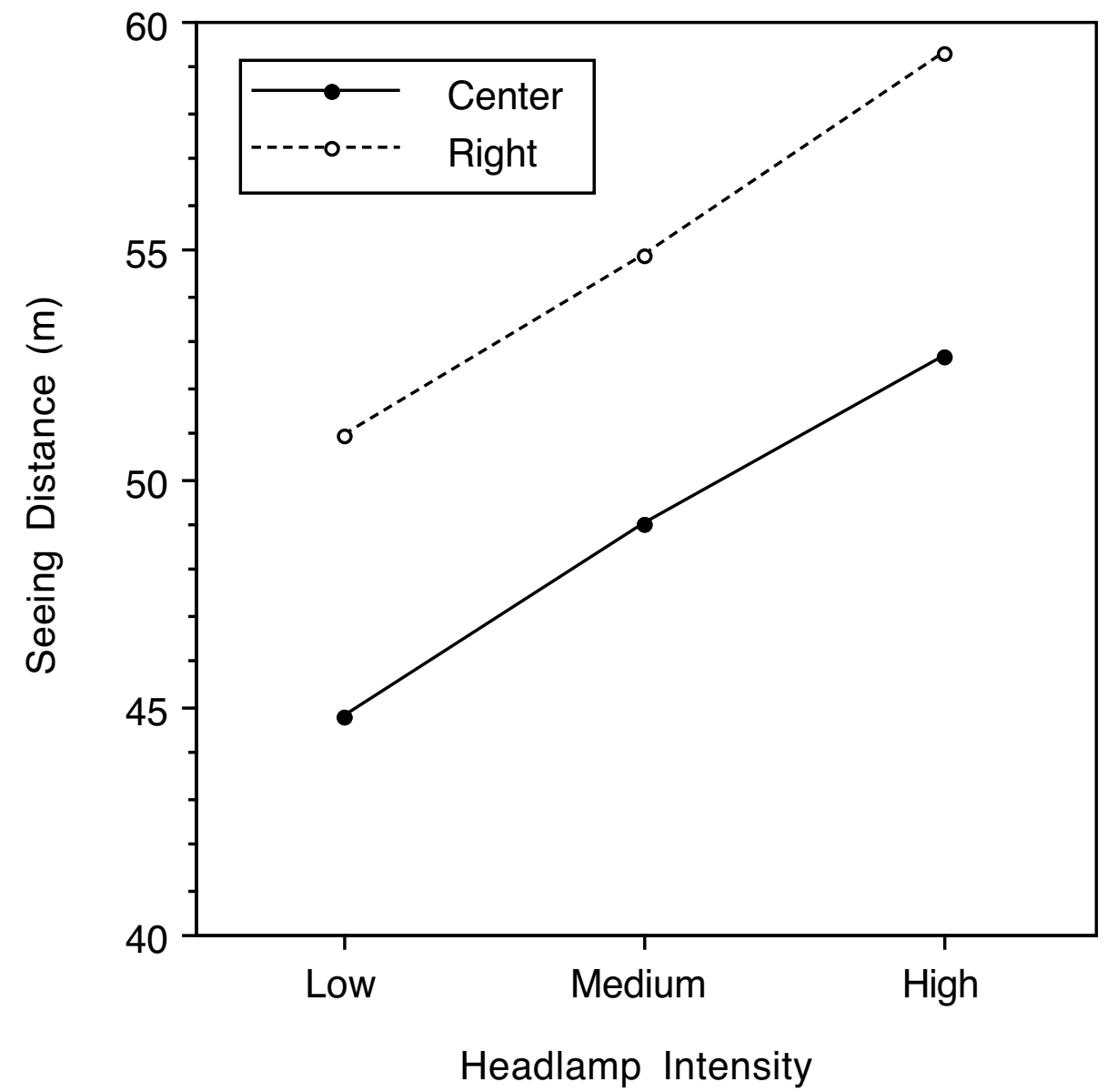

Figure 6. The effect of headlamp intensity on seeing distance for each path of the pedestrian. Seeing distances are longer when the pedestrian is on the right (further from the glare source in terms of visual angle). There is a strong effect of headlamp intensity for both paths. 


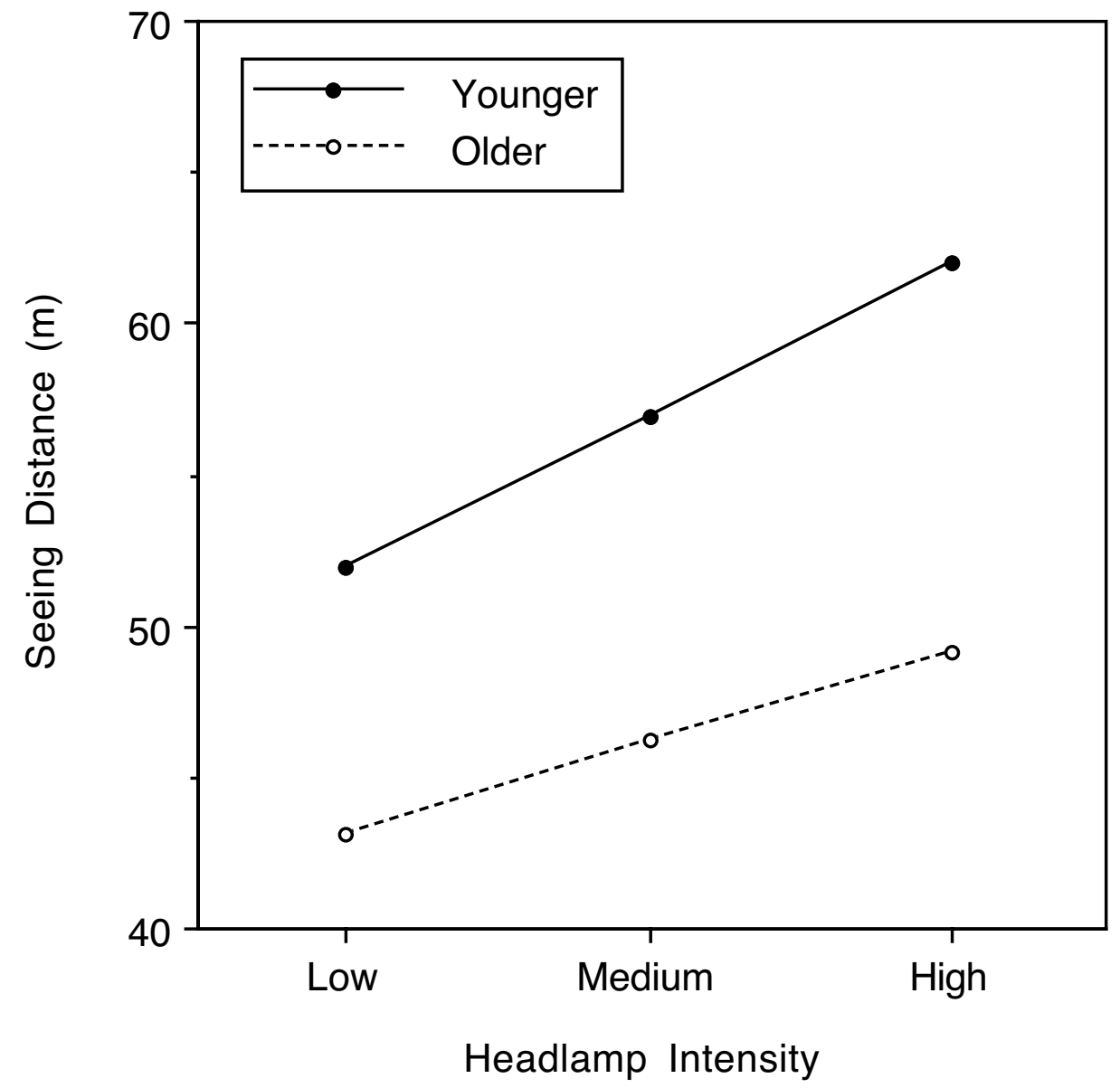

Figure 7. The effect of headlamp intensity on seeing distance for each age group. Seeing distances are longer for the younger subjects. For both age groups, seeing distances are longer with higher headlamp intensity. 


\section{Discomfort Ratings}

Although increased light resulted in longer seeing distances, the higher levels of glare produced more rated discomfort, as shown by the significant main effect of glare lamp intensity, $F(2,26)=59.6, p<.0001$ (see Figure 8). It made no difference whether the intensity of the seeing lamps was varied with the glare lamps or held at the minimum intensity; there was not a significant interaction of glare lamp intensity and seeing lamp intensity, $F(2,26)=0.41, p=.67$. The results were also the same across the two age groups. The interaction of glare lamp intensity and subject age group was not significant, $F(2,26)=0.11, p=.90$.

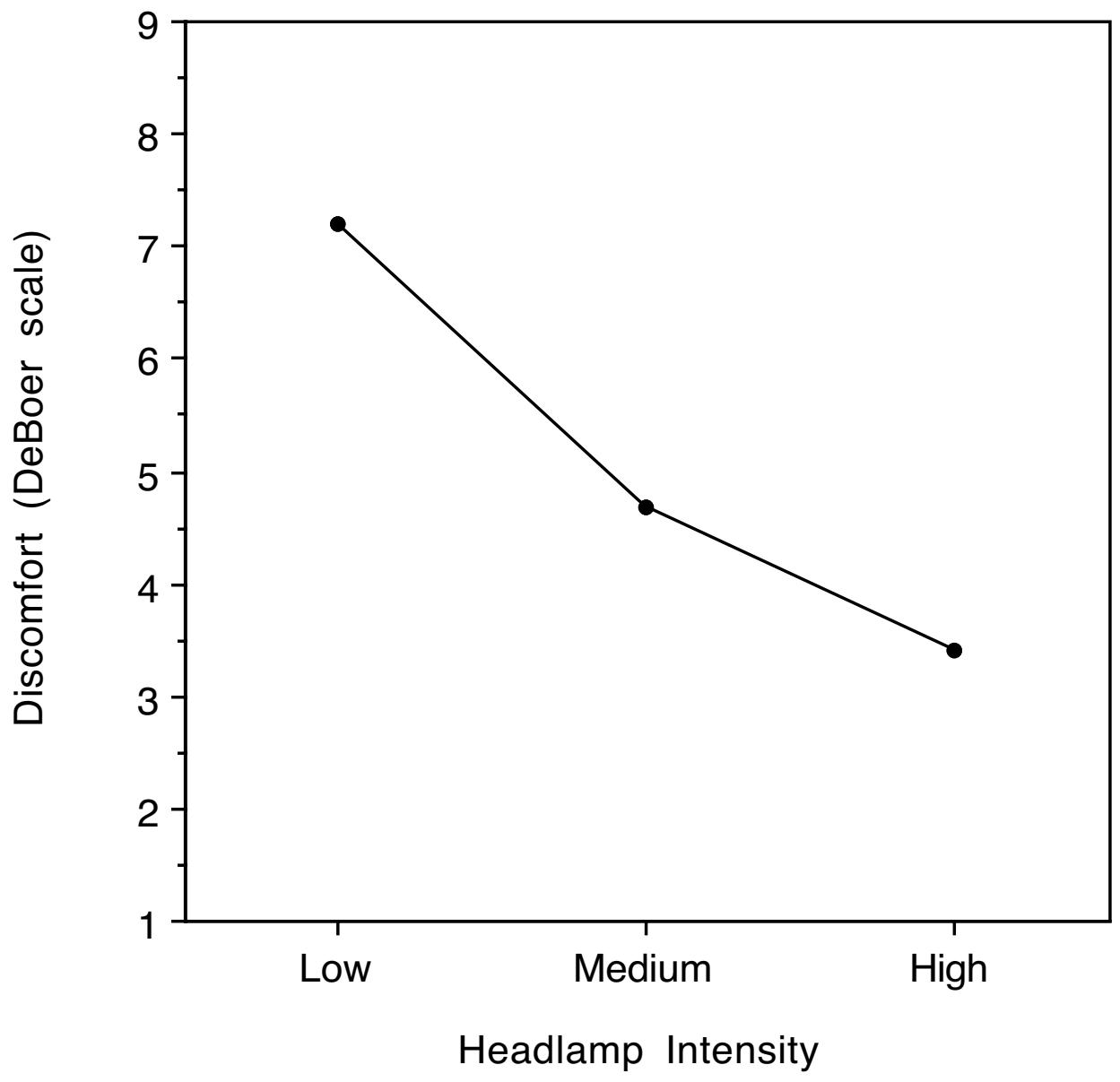

Figure 8. The effect of intensity on discomfort glare ratings. Lower numbers on the DeBoer scale correspond to more discomfort. 


\section{Discussion}

When the intensities of seeing lamps and glare lamps are varied proportionately, greater intensities result in substantially longer seeing distances. In the present experiment, increasing intensities by a factor of about 3.8 increased seeing distance by $17 \%$. The effect was about the same for younger and older people. This result is qualitatively consistent with earlier findings that seeing distance in a glare situation is longer when high beams oppose high beams than when low beams oppose low beams. It is also consistent with predictions from quantitative vision modeling using veiling luminance to represent the disabling effects of glare.

These findings suggest that, if objective visual performance is the only criterion, there is no clear upper limit to how intense headlamps should be. The increase in visual performance with higher intensities may break down at some level of intensity, but there is no indication in the present results of that happening. The range of intensities studied here extended up to about 3.8 times normal U.S. low beam intensities. This suggests that, even with no change in the relative distribution of light, higher light levels might produce safety benefits in night driving.

In the second part of the present experiment, discomfort glare ratings were found to increase with glare-lamp intensity. This is a reminder that objective visual performance is not necessarily the only criterion for an acceptable headlamp system. It may be that, although higher intensity headlamps increase seeing ability, there is a level at which people simply will not tolerate the discomforting effects of the glare. It is also possible that at some intensity people's behavior will change in ways that are not reflected in the task used here. For example, people may avert their gaze from the road ahead of them if the glare of oncoming headlamps is above a certain level. In the present experiment subjects had to look toward the glare source for only about 25 minutes, in contrast to the longer exposures that are sometimes necessary in night driving. Also, their motivation to perform well may have been higher than in normal driving because of a desire to cooperate with the experimenters. Furthermore, they did not have the workload of having to drive while they participated in the experiment.

Glare limits based solely on subjective discomfort may be high relative to the levels of glare that are currently common (with either U.S. or European-style lamps). One of the most comprehensive studies of the discomforting aspects of headlamp glare is work by Schmidt-Clausen and Bindels (1974). Based on that work, Sivak and Flannagan (1993) suggested that the discomforting aspects of glare, considered separately from the effects of glare on objective visual performance, led to a recommended maximum intensity of between 
810 and 2,478 cd (depending on the assumed level of adaptation luminance) at a point 0.25 degrees above and 2.0 degrees to the left of the straight ahead.

Although considerations other than objective seeing ability may be important in interpreting the present results, it is important to identify these considerations and recognize that they are, in fact, separate from objective seeing ability, and that taking them into account may result in lower visual performance than would otherwise be possible. Johansson, Bergstörm, et al. (1963) made the same point in commenting on their finding that seeing distances were longer with high beams in meeting situations:

The finding in these experiments...means that if visible distance on the near side of the road is taken as the only criterion of efficiency of meeting lights, dipping should never take place, or if it does it should take place later than is now customary....

[An] objection is that, although full headlights give greater visible distances, they cause so much discomfort from glare that they are intolerable under normal driving conditions. This may well be so, but if so it must be recognized that the gain of comfort resulting from dipping is obtained at the cost of some loss in visible distance, at any rate until the meeting cars are very close together. (pp. 178-179)

The argument presented here - that higher low-beam output results in substantially improved seeing distance-applies in its simplest form only to the unrealistic situation in which all vehicles' lamps would be changed at the same time. Because in reality it would take several years for a change in the vehicle population, increasing the intensities of new lamps would necessarily result in a period in which older vehicles would be exposed to more glare light (from the lamps of newer vehicles) without the benefit of more seeing light. However, a gradual transition, in which the total increase was accomplished in several smaller steps, might mitigate this problem. 


\section{Conclusions}

1. Increases in overall light output of current low-beam headlamps would result in increased seeing distance. How practical this might be as an approach to improving low-beam performance is limited by possible objective consequences of increased subjective discomfort levels, mismatches between the levels of older and newer lamps during a transition, and by the difficulties involved in generating and optically distributing substantially higher luminous flux levels.

2. Because subjective discomfort, rather than objective visual performance, may be the limiting consideration for setting maximum glare levels, more research should be done to understand the nature and consequences of discomfort glare. Because discomfort glare is subjective, it is a fundamentally more difficult research issue than objective visual performance. Research should explore the possibility that glare degrades objective seeing ability indirectly through effects on subjective discomfort. 


\section{References}

Bhise, V. D., Swigart, T. F., \& Farber, E. I. (1975). Development of a headlamp dimming request prediction model. In Proceedings of the Human Factors Society 19th Annual Meeting (pp. 289-295). Santa Monica: Human Factors Society.

Commission Internationale de l'Eclairage. (1981a). An analytic model for describing the influence of lighting parameters upon visual performance: Vol. 1. Technical foundations (Publication CIE 19/2.1). Paris: Author.

Commission Internationale de l'Eclairage. (1981b). An analytic model for describing the influence of lighting parameters upon visual performance: Vol. 2. Summary and application guidelines (Publication CIE 19/2.2). Paris: Author.

de Boer, J. B. (1967). Visual perception in road traffic and the field of vision of the motorist. In J. B. de Boer (Ed.) Public lighting (pp. 11-96). Eindhoven, The Netherlands: Philips Technical Library.

Holladay, L. L. (1926). The fundamentals of glare and visibility. Journal of the Optical Society of America and Review of Scientific Instruments, 12, 271-319.

Holladay, L. L. (1927). Action of a light source in the field of view in lowering visibility. Journal of the Optical Society of America and Review of Scientific Instruments, 14, $1-15$.

Johansson, G., Bergstörm, S.-S., Jansson, G., Ottander, C., Rumar, K., \& Örnberg, G. (1963). Visible distances in simulated night driving conditions with full and dipped headlights. Ergonomics, 6, 171-179.

Johansson, G., \& Rumar, K. (1963). Available braking distances in night driving (Report No. 13). Uppsala, Sweden: Department of Psychology, University of Uppsala.

Schmidt-Clausen, H.-J., \& Bindels, J. T. H. (1974). Assessment of discomfort glare in motor vehicle lighting. Lighting Research and Technology, 6, 79-88. 
Sivak, M., \& Flannagan, M. J. (1993). Partial harmonization of international standards for low-beam headlighting patterns (Report No. UMTRI-93-11). Ann Arbor: The University of Michigan Transportation Research Institute.

Sivak, M., \& Flannagan, M. J. (1994). Recent steps toward international harmonization of the low-beam headlamp pattern. International Journal of Vehicle Design, 15, 223 233.

Sivak, M., Helmers, G., Owens, D. A., \& Flannagan, M. (1992). Evaluation of proposed low-beam headlighting patterns (Report No. UMTRI-92-14). Ann Arbor: The University of Michigan Transportation Research Institute. 